\title{
CDISC SDTM Duchenne Muscular Dystrophy Findings About Test Name Terminology
}

National Cancer Institute

\section{Source}

National Cancer Institute. CDISC SDTM Duchenne Muscular Dystrophy Findings About

Test Name Terminology. NCI Thesaurus. Code C139019.

Terminology associated with the duchenne muscular dystrophy findings about test name codelist of the Clinical Data Interchange Standards Consortium (CDISC) Study Data Tabulation Model (SDT M). 